\title{
Serum levels of interleukin-17 and adiponectin are associated with infrapatellar fat pad volume and signal intensity alteration in patients with knee osteoarthritis
}

Kang Wang ${ }^{1,2}$, Jianhua $\mathrm{Xu}^{1}$, Jingyu Cai ${ }^{1}$, Shuang Zheng ${ }^{1}$, Weiyu Han ${ }^{2}$, Benny Antony ${ }^{2}$ and Changhai Ding ${ }^{1,2^{*}}$

\begin{abstract}
Background: In the present study, we sought to generate hypotheses regarding the associations of serum levels of interleukin (IL)-17, adiponectin, and resistin with magnetic resonance imaging-measured infrapatellar fat pad (IPFP) size and signal intensity alterations in patients with knee osteoarthritis (OA).

Methods: A total of 170 subjects with symptomatic knee OA (mean age 55.4 years, range $34-74,88.2 \%$ females) were included. IPFP volume was measured on T1-weighted spoiled gradient-recalled acquisition in the steady state images and was computed by using a software program. IPFP high signal intensity (grades 0-3) was assessed on T2-weighted fast spin echo images. Serum IL-17, adiponectin, and resistin levels were measured using an enzymelinked immunosorbent assay.

Results: In multivariable analyses, serum IL-17 was negatively associated with IPFP volume $(\beta=-0.185,95 \% \mathrm{Cl}-0$. 337 to -0.034$)$ but positively associated with the severity of IPFP signal intensity alteration (OR $1.23,95 \% \mathrm{Cl} 1.06-1$. 42) after adjustment for age, sex, weight, and height. Serum adiponectin was positively associated with IPFP volume ( $\beta=0.016,95 \% \mathrm{Cl} 0.001-0.032$ ) but negatively associated with IPFP signal intensity alteration (OR 0.99, $95 \% \mathrm{Cl} 0$. 98-1.00) after adjustment for covariates. Resistin was positively associated with IPFP signal intensity alteration (OR 1. $13,95 \%$ Cl 1.04-1.23) but not with IPFP volume. The significant associations of adiponectin or resistin disappeared after further adjustment for IL-17; in contrast, the significant associations of IL-17 remained after further adjustment for adiponectin.
\end{abstract}

Conclusions: While serum IL-17 and resistin were associated with reduced IPFP volume and/or increased abnormal signal intensity alteration, serum adiponectin had opposite associations that were largely through IL-17. These findings suggest that serum adipocytokines may have a role to play in IPFP changes of knee OA.

Keywords: Osteoarthritis, Infrapatellar fat pad, Adipokines, Cytokines, Cartilage, Epidemiology

Abbreviations: BMI, Body Mass Index; IL, Interleukin; ELISA, Enzyme-linked Immunosorbent Assay; IPFP, Infrapatellar Fat Pad; JSN, Joint Space Narrowing; K-L, Kellgren-Lawrence; MMP, Matrix Metalloproteinase; MRI, Magnetic Resonance Imaging; OA, Osteoarthritis; ROA, Radiographic Osteoarthritis; SPGR, Spoiled Gradient-recalled Acquisition in the Steady State; TNF, Tumor Necrosis Factor; VEGF, Vascular Endothelial Growth Factor

\footnotetext{
* Correspondence: changhai.ding@utas.edu.au

'Department of Rheumatology and Immunology, Arthritis Research Institute, the First Affiliated Hospital of Anhui Medical University, 218 Jixi Street, Hefei, China

${ }^{2}$ Menzies Institute for Medical Research, University of Tasmania, Private Bag

23, Hobart, Tasmania 7000, Australia
} 


\section{Background}

Osteoarthritis (OA) is a common joint disease with multiple pathogenetic mechanisms, affecting the whole joint. Obesity is considered one of the potent risk factors for developing OA [1]. Although the pathogenesis of OA is not well known, recent studies indicate that obesityrelated proinflammatory and metabolic factors contribute to OA progression [2]. Infrapatellar fat pad (IPFP), a local adipose tissue in knee joints with an abundance of adipocytes, immune cells, vessels, and nerve fibers, may be an active joint tissue involved in the initiation and progression of knee OA [3].

IPFP, also known as Hoffa's fat pad, is an intracapsular and extrasynovial structure [4]. It is thought to have primarily a biomechanical function of absorbing forces generated in the knee [5], and thus to have a protective effect physiologically. Indeed, in two recent studies, researchers reported that larger IPFP size was associated with reduced knee abnormal structural changes and symptoms [5, 6]. On the contrary, IPFP signal intensity alteration assessed using magnetic resonance imaging (MRI) was positively associated with the prevalence and/ or incidence of knee pain, cartilage defects, bone marrow lesions, and radiographic osteoarthritis (ROA) in older adults [7], and thus it may have a detrimental effect. IPFP has been considered an important source of cytokines and adipokines (i.e., adipocytokines) in OA [8]. IPFP tissues acquired from patients with knee OA can produce cytokines such as interleukin (IL)-1 $\beta$, tumor necrosis factor (TNF)- $\alpha$, IL-6, IL-8, IL-17, basic fibroblast growth factor, and vascular endothelial growth factor (VEGF) [8-10]. It can also produce various adipokines such as leptin, resistin, and adiponectin [11]. Researchers in an in vitro study reported that conditioned media from cultured white adipose tissue from OA IPFP that contained leptin could induce cartilage collagen release and increase matrix metalloproteinase (MMP)-1 and MMP-13 expression in chondrocytes, and thus had catabolic effects on cartilage [12].

Although the local inflammatory profile of IPFP tissue has been investigated in vitro, the associations between serum adipocytokines and IPFP changes have not been examined in epidemiological or clinical studies. Osteoarthritic IPFP explants can release high protein levels of IL-17, resistin, and adiponectin [10]. While serum IL-17 and resistin (proinflammatory adipocytokines) concentrations were significantly higher in patients with knee OA than in control subjects [13], serum levels of adiponectin (an anti-inflammatory adipocytokine) were associated with decreased disease severity of knee OA [14]. It is unknown if IPFP measures such as size and signal intensity alteration are associated with increased or decreased release of systemic IL-17, resistin, and adiponectin in knee OA. We expected that IPFP size would be associated with decreased levels of serum IL-17 and resistin and an increased level of serum adiponectin, but IPFP high signal intensity would be associated with increased levels of serum IL-17 and resistin and a decreased level of serum adiponectin in patients with knee OA. The aim of this study was therefore to generate hypotheses regarding the associations between serum levels of IL-17, adiponectin, and resistin and the volume and signal intensity alteration of IPFP measured using MRI in patients with symptomatic knee OA.

\section{Methods \\ Study design and patients}

We consecutively enrolled into the Anhui Osteoarthritis Study 205 patients with OA who fulfilled the American College of Rheumatology criteria for the classification of clinical knee OA at the Outpatient Clinics, Department of Rheumatology, First Affiliated Hospital of Anhui Medical University, from January 2012 to November 2013. We excluded institutionalized persons, patients with rheumatoid arthritis or other inflammatory diseases, patients with severe knee OA who were planning to have knee arthroplasty in 2 years (this study was ongoing with 2 years of follow-up), and patients with contraindications to MRI (including metal sutures, presence of shrapnel, iron filings in the eye, and claustrophobia). The study was approved by the First Affiliated Hospital of Anhui Medical University Ethics Committee, and all participants signed the informed consent forms.

\section{Anthropometrics}

Weight was measured to the nearest $0.1 \mathrm{~kg}$ (with shoes, socks, and bulky clothing removed) using a single pair of electronic scales (RGZ-120; Jiangsu Province, China) that were calibrated using a known weight at the beginning of each clinic. Height was measured to the nearest $0.1 \mathrm{~cm}$ by using a stadiometer with shoes, socks, and headgear removed. Body mass index (BMI) (weight [kg]/ height $\left.\left[\mathrm{m}^{2}\right]\right)$ was calculated.

\section{Radiographic OA assessment}

All patients underwent knee radiography. The 15-degree flexion, standing, anteroposterior view image was taken in the symptomatic knees (the severer one if both knees were affected; the right one if both knees were equally painful). Radiographic assessment was performed by a radiology specialist using the Kellgren-Lawrence (K-L) grading system (grades $0-4)$ : grade 0 , normal; grade 1 , no joint space narrowing (JSN), suspicious osteophytes; grade 2, suspicious JSN, mild osteophytes; grade 3, definite JSN, moderate osteophytes, and/or subchondral bone sclerosis; and grade 4, marked JSN, large osteophytes, and/or severe subchondral bone sclerosis [15]. Radiographic OA was defined as a $\mathrm{K}-\mathrm{L}$ grade $\geq 2$. 
Assessments of IPFP volume and signal intensity changes MRI of the selected knee was performed with a 3.0-T whole-body magnetic resonance imaging unit (Signa HDxT 3.0T; GE Healthcare, Little Chalfont, UK), using a commercial transmit/receive extremity coil. The following sequence and parameters were used:

1. A T1-weighted fat saturation three-dimensional spoiled gradient-recalled acquisition in the steady state (SPGR), flip angle 30 degrees; repetition time 31 milliseconds; echo time 6.71 milliseconds; field of view $16 \mathrm{~cm}$; 60 partitions; $512 \times 512$-pixel matrix; acquisition time 11 minutes, 56 milliseconds; and 1 acquisition (Sagittal images were obtained at a partition thickness of $1.5 \mathrm{~mm}$ and an in-plane resolution of $0.31 \times 0.31$ [ $512 \times 512$ pixels].)

2. A T2-weighted fat saturation two-dimensional fast spin echo, flip angle 90 degrees, repetition time 3067 milliseconds, echo time 112 milliseconds, field of view $16 \mathrm{~cm}, 15$ partitions, $256 \times 256$-pixel matrix (Sagittal images were obtained at a slice thickness of $4 \mathrm{~mm}$ with an interslice gap of $1.0 \mathrm{~mm}$. Images were checked for image noise and structural abnormalities interfering with segmentation.)

IPFP area was measured by manually drawing disarticulation contours around the IPFP boundaries on T1weighted SPGR MRI scans using OsiriX software (Pixmeo Sàrl, Bernex, Switzerland). IPFP volume was computed by using the software program. IPFP signal intensity alteration on T2-weighted MRI studies was recorded if hyperintense signal alterations were observed within the IPFP. Signal intensity alteration, defined as discrete areas of increased signal within the IPFP, was graded as follows: grade 0 , none; grade $1,<10 \%$ of the region; grade $2,10-$ $20 \%$ of the region; and grade $3,>20 \%$ of the region [16]. All images were grouped together and read in randomized order by two readers, with the reader blinded to subjects' information. Intraobserver reliability was measured in 30 subjects, with an intraclass correlation coefficient of 0.95 (95\% CI 0.89-0.97). Interobserver reliability was 0.94 (95\% CI 0.85-0.97).

\section{Serum adipocytokine measurements}

Morning fasting blood samples were collected from patients. Serum was separated, aliquoted into plastic storage tubes, and stored at $-80{ }^{\circ} \mathrm{C}$ until analysis. Serum levels of IL-17, adiponectin, and resistin were measured by enzyme-linked immunosorbent assay (ELISA) (eBioscience, San Diego, CA, USA) kits according to the manufacturer's instructions. The optical density was measured at $450 \mathrm{~nm}$ using an automatic ELISA reader (Sunrise; Tecan, Männedorf, Switzerland). The limits of detection for IL-17, adiponectin, and resistin were
$0.5 \mathrm{pg} / \mathrm{ml}, 0.01 \mathrm{ng} / \mathrm{ml}$, and $3.1 \mathrm{pg} / \mathrm{ml}$, respectively, and the coefficients of variation were $7.1 \%, 4.2 \%$, and $5.1 \%$, respectively.

\section{Data analysis}

Student's $t$ test, the $\chi^{2}$ test, and the Mann-Whitney $U$ test was used to compare means, proportions, and medians, respectively. Pearson's correlations or Spearman's analyses were used to analyze the correlations of serum levels of IL-17 (log-transformed) with serum levels of adiponectin and resistin (log-transformed) or K-L grading. Linear regression analyses were used to examine the associations between IL-17, adiponectin, or resistin and IPFP volume (the dependent variable that was normally distributed) before and after adjustment for covariates including age, sex, weight, and height. Ordinal regression analyses were used to examine the associations between IL-17, adiponectin, or resistin and IPFP signal intensity alteration (the dependent variable). Standard diagnostic checks of model fit and residuals were routinely done, and data points with large residuals and/or high influence were investigated for data errors. A $p$ value $<0.05$ (two-tailed) or a $95 \% \mathrm{CI}$ not including the null point was regarded as statistically significant. All statistical analyses were performed using SPSS 13.0 for Windows (SPSS, Chicago, IL, USA).

\section{Results}

A total of 170 subjects ( $88.2 \%$ females) aged between 34 and 74 years (mean 55.4 years) were included in the analyses, and another 35 subjects were excluded from the study because of incomplete data. There were no significant differences in demographic factors between those included and excluded (data not shown). The median IL-17 level was $2.00 \mathrm{pg} / \mathrm{ml}$, the mean IPFP volume was $20.46 \mathrm{ml}$ (range 12.39-42.90 ml), and the IPFP signal intensity alteration mean value was 0.78 (range $0-3$ ). There were $73 \%$ patients who had established ROA.

Characteristics of the subjects based on the median value of IL-17 are presented in Table 1. Patients with higher and lower levels of IL-17 were similar in terms of sex, BMI, height, weight, knee OA, IPFP signal intensity alteration, IPFP volume, and resistin level. However, patients with higher levels of IL-17 were older and had lower adiponectin levels. Serum levels of IL-17 were significantly associated with serum levels of adiponectin $(r=-0.234, p=0.002)$ and resistin $(r=0.165, p=0.032)$. Serum IL-17 and resistin were not associated with K-L grade, but adiponectin was negatively correlated with $\mathrm{K}$ $\mathrm{L}$ grade $(\rho=-0.211, p=0.006)$.

Serum level of IL-17 was significantly and negatively associated with IPFP volume after adjustment for age, sex, weight, and height (Table 2, Fig. 1), and this association remained unchanged after further adjustment for 
Table 1 Characteristics of participants (split by median level of interleukin 17)

\begin{tabular}{|c|c|c|c|c|}
\hline & Total $(n=170)$ & $\mathrm{IL}-17 \leq$ median $(n=85)$ & IL-17 > median $(n=85)$ & $p$ Values \\
\hline Age, years $^{a}$ & $55.45(8.25)$ & $54.07(8.17)$ & $56.66(8.39)$ & 0.044 \\
\hline Female sex, $\%^{\mathrm{b}}$ & 88.2 & 89 & 87 & 0.634 \\
\hline Height, $\mathrm{cm}^{\mathrm{a}}$ & $159.00(6.89)$ & $159.09(7.01)$ & $158.58(6.88)$ & 0.638 \\
\hline Weight, $\mathrm{kg}^{\mathrm{a}}$ & $64.83(10.56)$ & $64.23(9.62)$ & $65.09(10.70)$ & 0.591 \\
\hline $\mathrm{BMl}, \mathrm{kg} / \mathrm{m}^{2 a}$ & $25.64(3.85)$ & $25.35(3.20)$ & $25.87(3.88)$ & 0.341 \\
\hline K-L grade, $\%^{\mathrm{b}}$ & & & & 0.268 \\
\hline 1 & 27.9 & 25.7 & 28.6 & \\
\hline 2 & 41.9 & 50.0 & 35.1 & \\
\hline 3 & 25.7 & 20.3 & 31.2 & \\
\hline 4 & 4.5 & 4.1 & 5.2 & \\
\hline Knee ROA, \% ${ }^{\mathrm{b}}$ & 72.1 & 74.3 & 71.4 & 0.689 \\
\hline IPFP signal intensity alteration, $\%^{\mathrm{b}}$ & 43.1 & 44.2 & 40 & 0.298 \\
\hline IPFP volume, mm $\mathrm{m}^{3 \mathrm{a}}$ & $20.46(5.01)$ & $20.57(5.53)$ & $20.42(4.88)$ & 0.855 \\
\hline Adiponectin, pg/ml ${ }^{\mathrm{C}}$ & $18.67(3.94-53.07)$ & $35.08(11.36-71.26)$ & $12.82(3.00-46.94)$ & 0.001 \\
\hline Resistin, $\mathrm{ng} / \mathrm{ml}^{\mathrm{c}}$ & $2.37(1.40-5.57)$ & $2.18(1.45-4.86)$ & $2.81(1.32-5.78)$ & 0.532 \\
\hline
\end{tabular}

Abbreviations: $B M I$ body mass index, $K$-L Kellgren-Lawrence, IL interleukin, $R O A$ radiographic osteoarthritis, IPFP infrapatellar fat pad

$\mathrm{ROA}$ was defined as a K-L grade $\geq 2$. IL-17 median level $2.00 \mathrm{pg} / \mathrm{ml}$ (interquartile range $1.56-2.82$ ). Data in bold denote statistically significant results

${ }^{\mathrm{a}} t$ tests were used for mean (SD)

${ }^{b} X^{2}$ tests were used for the proportions

${ }^{c}$ Mann-Whitney $U$ tests were used for median (interquartile range)

adiponectin (Table 2). Serum level of adiponectin was significantly and positively associated with IPFP volume after adjustment for age, sex, weight, and height, but this became nonsignificant after further adjustment for IL-17 (Table 2). There was no significant association between resistin and IPFP volume before or after adjustment for potential confounders.

Serum levels of IL-17 were significantly and positively associated with severity of IPFP signal intensity alteration before and after adjustment for age, sex, weight, and height, and this association remained significant after further adjustment for adiponectin (Fig. 2, Table 3). Adiponectin had a negatively significant and resistin a positively significant association with IPFP signal intensity alteration before and after adjustment for age, sex, weight, and height, but these associations became nonsignificant after further adjustment for IL-17 (Table 3).

The significant associations of IL-17 and resistin remained significant, but associations of adiponectin became nonsignificant, after adjustment for $\mathrm{K}-\mathrm{L}$ grade (data not shown).

\section{Discussion}

To our knowledge, this is the first epidemiological study to illustrate the relationship between serum levels of adipocytokines and IPFP volume and signal intensity alteration in patients with knee OA. After adjustment for age, sex, weight, and height, we found that higher serum IL-17 level was associated with lower IPFP volume and increased IPFP signal intensity alteration (indicating poorer IPFP quality). Higher adiponectin level was associated with higher IPFP volume and better IPFP quality, but higher serum resistin level was associated only with poorer IPFP quality. These suggest that serum levels of adipocytokines are associated with IPFP pathophysiology in knee OA.

The role of IPFP in OA progression is inconclusive. In our previous studies, we have reported that IPFP size may have a protective role in knee OA structural

Table 2 Association between adipocytokines and infrapatellar fat pad volume

\begin{tabular}{|c|c|c|c|c|}
\hline Adipocytokines & Multivariable $\beta$ (95 \% Cl) & $p$ Value & Multivariable $\beta$ (95 \% Cl) & $p$ Value \\
\hline $\mid \mathrm{L}-17$ & $-0.19(-0.34$ to -0.03$)$ & 0.017 & $-0.16(-0.32 \text { to }-0.01)^{b}$ & 0.047 \\
\hline Adiponectin & 0.02 (0.01 to 0.03$)$ & 0.039 & $0.01(-0.01 \text { to } 0.03)^{c}$ & 0.190 \\
\hline Resistin & $-0.07(-0.20$ to 0.06$)$ & 0.303 & $0.02(-0.01 \text { to } 0.18)^{c}$ & 0.773 \\
\hline
\end{tabular}

\section{IL interleukin}

Dependent variable: infrapatellar fat pad volume. Independent variable: cytokines. Data in bold denote statistically significant results

${ }^{a}$ Adjusted for age, sex, weight and height

${ }^{\mathrm{b}}$ Further adjustment for adiponectin

${ }^{\mathrm{C}}$ Further adjustment for IL-17 


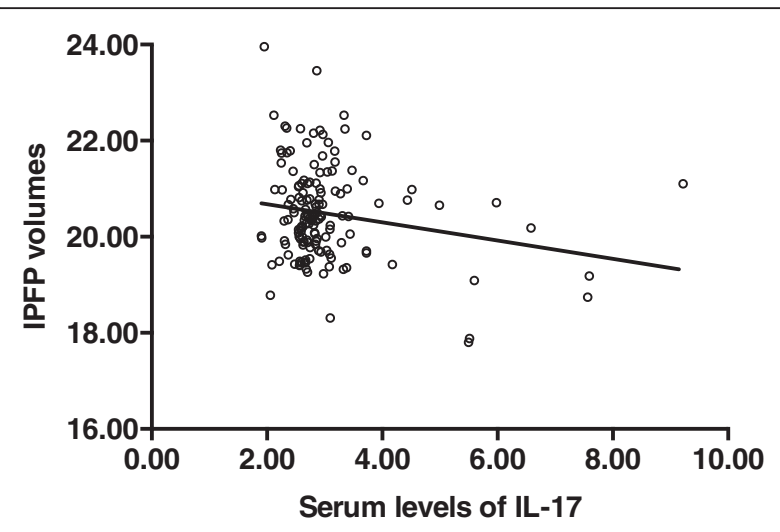

Fig. 1 Association between serum IL-17 and infrapatellar fat pad volume in patients with knee osteoarthritis. $r=-0.199, p=0.015$ after adjustment for age, sex, height and weight. IL interleukin, IPFP infrapatellar fat pad

changes: Greater IPFP maximal area is associated with greater cartilage volume and lesser cartilage defects, bone marrow lesions, and R OA cross-sectionally and/or longitudinally in older adults $[5,6]$. Although researchers in one study reported that IPFP volume was correlated only with age in patients with knee OA and might not be related to the progression of OA [17], we recently reported that, in patients with knee OA, IPFP volume was associated with greater cartilage volume and fewer cartilage defects, bone marrow lesions, and osteophytes [18]. The signal intensity changes in IPFP were positively associated with the prevalence and/or incidence of knee pain, cartilage defects, bone marrow lesions, and $\mathrm{R} \mathrm{OA}$ in older adults [7], suggesting that abnormal quality of IPFP is detrimental for knee symptoms and structures. This is supported by a recent study demonstrating that synovitis

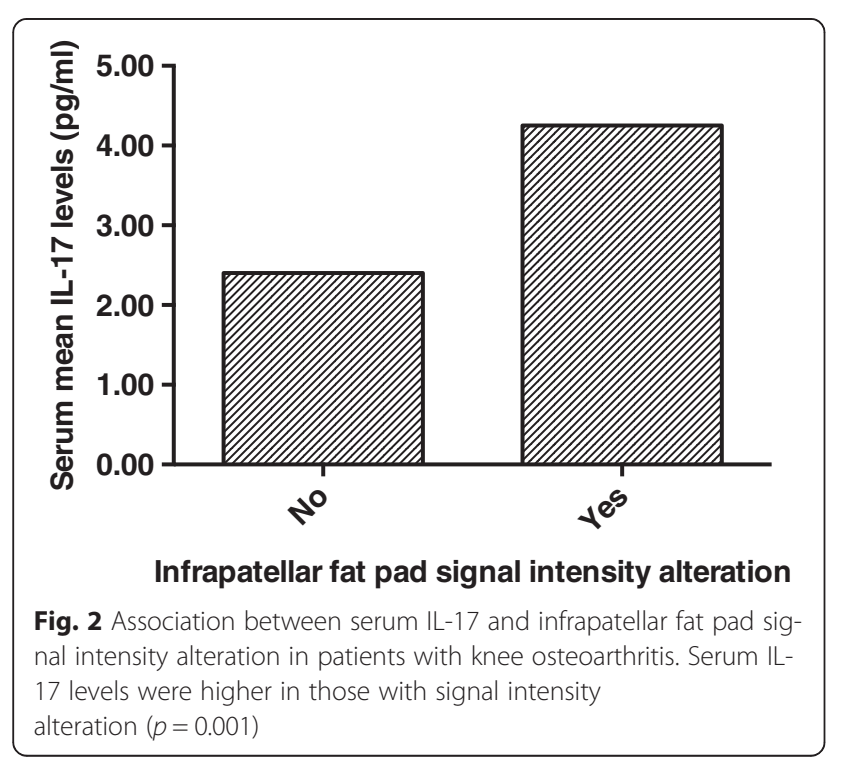

measured using IPFP signal intensity change (Hoffa's synovitis) is associated with the development of radiographic knee OA [19]. The severity of inflammation in IPFP measured by dynamic contrast-enhanced MRI was associated with the severity of pain in knee OA [20].

Animal or in vitro studies have indicated a potentially important role of IPFP in mediating knee intra-articular inflammation. IPFP produced an elevated level of inflammatory cytokines, growth factors, and adipokines in a high-fat diet-induced murine OA model, and the expression levels of the adipokines were significantly correlated with expression of TNF- $\alpha$, VEGF, and transforming growth factor $\beta$ [21]. Rates of IL-6 expression and secretion were higher in IPFP tissue than in subcutaneous adipose tissue, indicating that the IPFP cytokine profile could play a role in paracrine inflammation via the local production of IL- 6 and contribute to damage in adjacent cartilage in patients with OA [8]. IPFP explants from patients with OA could produce a variety of cytokines, and the production was increased by local cytokine stimulation [9].

Preliminary evidence has shown that adipocytokines such as IL-17 may have roles to play in knee OA. A significant proportion (up to $20 \%$ ) of chondrocytes in cartilage samples from patients with OA expressed IL-17R [22]. An in vitro study suggested that IL-17 induced the release of chemokines by chondrocytes and synovial fibroblasts, contributing to cartilage breakdown and synovial infiltration in OA [23]. Researchers in a crosssectional study reported that serum IL-17 concentrations were significantly higher in patients with knee OA than in control subjects, and synovial IL-17 concentrations were positively correlated with K-L grade and Lequesne index in patients with knee OA [24]. So far, there have been no studies illustrating the relationship between serum IL-17 levels and IPFP changes in patients with knee OA. We found that serum level of IL-17 was negatively associated with IPFP volume and positively with IPFP high signal intensity in patients with knee OA. According to the biopsies from the infrapatellar region in knee OA, chronic low-grade inflammation was detected as signal changes on fluid-sensitive MRI sequences [25]. Our results suggest that serum IL-17 is associated with IPFP pathophysiology, but it is unknown if IL-17 contributes to IPFP inflammation or if abnormal IPFP may produce IL-17, which could play a role in OA pathogenesis. The causal relationship needs to be confirmed in future longitudinal studies.

The role of adiponectin in OA is largely unclear. In vitro findings suggested that adiponectin increased nitric oxide and MMP production in human OA chondrocytes mainly via the AMP-activated protein kinase/c-Jun Nterminal kinase pathway, which would lead to accelerated degradation of OA cartilage matrix ex vivo [26]. Adiponectin can also induce IL-6 secretion in a cultured 
Table 3 Association between adipocytokines and infrapatellar fat pad signal intensity alteration

\begin{tabular}{|c|c|c|c|c|}
\hline Adipocytokines & Multivariable $\mathrm{aR}(95 \% \mathrm{Cl})$ & $p$ Value & Multivariable OR (95 \% Cl) & $p$ Value \\
\hline IL-17 & $1.23(1.06-1.42)$ & 0.007 & $1.20(1.03-1.38)^{b}$ & 0.017 \\
\hline Adiponectin & $0.99(0.98-1.00)$ & 0.042 & $0.99(0.98-1.00)^{c}$ & 0.177 \\
\hline Resistin & $1.13(1.04-1.23)$ & 0.004 & $1.11(1.00-1.23)^{c}$ & 0.054 \\
\hline
\end{tabular}

IL interleukin

Dependent variables: IPFP signal intensity alteration (0-3). Independent variable: adipocytokines. Data in bold denote statistically significant results

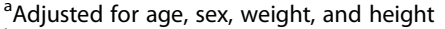

${ }^{b}$ Further adjustment for adiponectin

${ }^{\mathrm{C}}$ Further adjustment for IL-17

chondrogenic cell line [27]. In contrast, adiponectin levels in both plasma and synovial fluid decreased significantly as the severity of OA (evaluated by K-L grading) increased in humans [28]. Serum adiponectin levels were negatively associated with radiographic progression in patients with hand OA $[29,30]$. Furthermore, adiponectin expression in IPFP was higher than in subcutaneous adipose tissue in patients with knee OA [8] and was higher in patients with $\mathrm{OA}$ in end stage than in early stage [31]. In our present study, the serum levels of adiponectin were positively associated with IPFP volume and negatively with IPFP signal intensity alteration, indicating that adiponectin may have a role in IPFP of knee OA. This association was dependent on IL-17 and K-L grade, suggesting that this may be mediated largely by IL-17 or ROA.

The biological effects of resistin in OA remain controversial. Recently, researchers in one study reported that resistin could upregulate the expression of multiple chemokines, cytokines, and matrix-degrading genes that are involved in OA pathobiology [32]. Researchers in a cross-sectional study reported that serum resistin levels in patients with hand ROA were higher than in patients with nonradiographic hand OA and control subjects, and were associated with radiographic bone erosion in hand OA [33]. In the present study, we did not find a significant association between resistin and IPFP volume, but we found a significantly positive association between resistin and IPFP signal intensity alteration, suggesting a potential role of resistin in abnormal IPFP changes of knee OA.

The present study has some limitations. First, it was a cross-sectional study designed to generate hypotheses, and the causal relationship cannot be interpreted. Second, the subjects were recruited from the clinics consecutively rather than from the community randomly, so the results may not generalizable to patients with general knee OA. Third, the signal intensity alteration of IPFP was assessed by unenhanced MRI, which was nonspecific, and its pathology is largely unclear [34]. Last, the levels of adipocytokines were measured in serum rather than in synovial fluid, so their local effects are unknown.

\section{Conclusions}

While serum IL-17 and resistin were associated with reduced IPFP volume and/or increased abnormal signal intensity alteration, serum adiponectin had opposite associations, which were largely through IL-17. These results suggest that serum levels of adipocytokines may have a role to play in IPFP changes of knee OA.

\section{Acknowledgements \\ Special thanks go to the subjects who made this study possible. We also thank Hui Zhang for technical support.}

\section{Funding}

This study was supported by the National Natural Science Foundation of China (81172865).

\section{Authors' contributions}

KW had full access to all the study and took responsibility for the integrity of the data and accuracy of the statistical analyses. CD, JX, and KW designed the study. KW, JX, JC, SZ, WH, and BA acquired data. KW and CD interpretation data. KW, JX, JC, SZ, WH, BA, and CD prepared and approved the manuscript.

\section{Authors' information}

$C D$ 's research interests center on epidemiological and clinical investigation of osteoarthritis using modern techniques such as magnetic resonance imaging. He is looking at the inflammatory and metabolic mechanisms of osteoarthritis and osteoporosis, and he is interested in evaluating new therapies for osteoarthritis and other inflammatory diseases. He has received grants from competitive sources totaling more than $\$ 4$ million, and he has published over 150 manuscripts in international peer-reviewed journals with an $h$-index of 35 .

\section{Competing interests}

The authors declare that they have no competing interests.

\section{Ethics approval and consent to participate}

The study was approved by Anhui Medical University Medical Ethics Committee, and written informed consent was obtained from all participants.

Received: 13 May 2016 Accepted: 3 August 2016

Published online: 26 August 2016

\section{References}

1. Grotle M, Hagen KB, Natvig B, Dahl FA, Kvien TK. Obesity and osteoarthritis in knee, hip and/or hand: an epidemiological study in the general population with 10 years follow-up. BMC Musculoskel Dis. 2008;9:132.

2. Wang $X$, Hunter $D, X u$ J, Ding C. Metabolic triggered inflammation in osteoarthritis. Osteoarthritis Cartilage. 2015;23:22-30.

3. Clockaerts S, Bastiaansen-Jenniskens YM, Runhaar J, Van Osch GJ, Van Offel JF, Verhaar JA, et al. The infrapatellar fat pad should be considered as an active osteoarthritic joint tissue: a narrative review. Osteoarthritis Cartilage. 2010;18:876-82. 
4. Saddik D, McNally EG, Richardson M. MRI of Hoffa's fat pad. Skeletal Radiol. 2004;33:433-44.

5. Han W, Cai S, Liu Z, Jin X, Wang X, Antony B, et al. Infrapatellar fat pad in the knee: is local fat good or bad for knee osteoarthritis? Arthritis Res Ther. 2014;16:R145.

6. Pan F, Han W, Wang X, Liu Z, Jin X, Antony B, et al. A longitudinal study of the association between infrapatellar fat pad maximal area and changes in knee symptoms and structure in older adults. Ann Rheum Dis. 2015;74:1818-24.

7. Han W, Aitken D, Zhu Z, Halliday A, Wang X, Antony B, et al. Signal intensity alteration in the infrapatellar fat pad at baseline for the prediction of knee symptoms and structure in older adults: a cohort study. Ann Rheum Dis. 2015. doi:10.1136/annrheumdis-2015-208360.

8. Klein-Wieringa IR, Kloppenburg M, Bastiaansen-Jenniskens YM, Yusuf E, Kwekkeboom JC, El-Bannoudi $\mathrm{H}$, et al. The infrapatellar fat pad of patients with osteoarthritis has an inflammatory phenotype. Ann Rheum Dis. 2011; 70:851-7.

9. Distel E, Cadoudal T, Durant S, Poignard A, Chevalier X, Benelli C. The infrapatellar fat pad in knee osteoarthritis: an important source of interleukin-6 and its soluble receptor. Arthritis Rheum. 2009;60:3374-7.

10. Clockaerts S, Bastiaansen-Jenniskens YM, Feijt C, De Clerck L, Verhaar JA, Zuurmond AM, et al. Cytokine production by infrapatellar fat pad can be stimulated by interleukin $1 \beta$ and inhibited by peroxisome proliferator activated receptor a agonist. Ann Rheum Dis. 2012;71:1012-8.

11. Eymard F, Pigenet A, Citadelle D, Flouzat-Lachaniette $\mathrm{CH}$, Poignard A, Benelli $C$, et al. Induction of an inflammatory and prodegradative phenotype in autologous fibroblast-like synoviocytes by the infrapatellar fat pad from patients with knee osteoarthritis. Arthritis Rheumatol. 2014; 66:2165-74

12. Hui W, Litherland GJ, Elias MS, Kitson GI, Cawston TE, Rowan AD, et al. Leptin produced by joint white adipose tissue induces cartilage degradation via upregulation and activation of matrix metalloproteinases. Ann Rheumatic Dis. 2012;71:455-62

13. de Boer TN, van Spil WE, Huisman AM, Polak AA, Bijlsma JW, Lafeber FP, et al. Serum adipokines in osteoarthritis; comparison with controls and relationship with local parameters of synovial inflammation and cartilage damage. Osteoarthritis Cartilage. 2012;20:846-53.

14. Zheng $\mathrm{S}, \mathrm{Xu}$ J, Xu S, Zhang M, Huang S, He F, et al. Association between circulating adipokines, radiographic changes, and knee cartilage volume in patients with knee osteoarthritis. Scand J Rheumatol. 2016;45:224-9.

15. Kellgren JH, Lawrence JS. Radiological assessment of osteoarthrosis. Ann Rheum Dis. 1957;16:494-502.

16. Hunter DJ, Guermazi A, Lo GH, Grainger AJ, Conaghan PG, Boudreau RM, et al. Evolution of semi-quantitative whole joint assessment of knee OA: MOAKS (MRI Osteoarthritis Knee Score). Osteoarthritis Cartilage. 2011;19:990-1002.

17. Chuckpaiwong B, Charles HC, Kraus VB, Guilak F, Nunley JA. Age-associated increases in the size of the infrapatellar fat pad in knee osteoarthritis as measured by 3 T MRI. J Orthop Res. 2010;28:1149-54.

18. Cai J, Xu J, Wang K, Zheng S, He F, Huan S, et al. Association between infrapatellar fat pad volume and knee structural changes in patients with knee osteoarthritis. J Rheumatol. 2015;42:1878-84.

19. Roemer FW, Kwoh CK, Hannon MJ, Hunter DJ, Eckstein F, Fujii T, et al. What comes first? Multitissue involvement leading to radiographic osteoarthritis: magnetic resonance imaging-based trajectory analysis over four years in the Osteoarthritis Initiative. Arthritis Rheumatol. 2015;67:2085-96.

20. Ballegaard C, Riis RG, Bliddal $H$, Christensen R, Henriksen M, Bartels EM, et al. Knee pain and inflammation in the infrapatellar fat pad estimated by conventional and dynamic contrast-enhanced magnetic resonance imaging in obese patients with osteoarthritis: a cross-sectional study. Osteoarthritis Cartilage. 2014;22:933-40.

21. Iwata M, Ochi H, Hara Y, Tagawa M, Koga D, Okawa A, et al. Initial responses of articular tissues in a murine high-fat diet-induced osteoarthritis model: pivotal role of the IPFP as a cytokine fountain. PLoS One. 2013;8:e60706.

22. Honorati MC, Meliconi R, Pulsatelli L, Canè S, Frizziero L, Facchini A. High in vivo expression of interleukin-17 receptor in synovial endothelial cells and chondrocytes from arthritis patients. Rheumatology. 2001;40:522-7.

23. Honorati MC, Bovara M, Cattini L, Piacentini A, Facchini A. Contribution of interleukin 17 to human cartilage degradation and synovial inflammation in osteoarthritis. Osteoarthritis Cartilage. 2002;10:799-807.

24. Chen B, Deng Y, Tan Y, Qin J, Chen LB. Association between severity of knee osteoarthritis and serum and synovial fluid interleukin 17 concentrations. J Int Med Res. 2014;42:138-44.
25. Fernandez-Madrid F, Karvonen RL, Teitge RA, Miller PR, An T, Negendank WG Synovial thickening detected by MR imaging in osteoarthritis of the knee confirmed by biopsy as synovitis. Magn Reson Imaging. 1995;13:177-83.

26. Kang EH, Lee YJ, Kim TK, Chang CB, Chung JH, Shin K, et al. Adiponectin is a potential catabolic mediator in osteoarthritis cartilage. Arthritis Res Ther. 2010;12:R231.

27. Lago R, Gomez R, Otero M, Lago F, Gallego R, Dieguez C, et al. A new player in cartilage homeostasis: adiponectin induces nitric oxide synthase type II and pro-inflammatory cytokines in chondrocytes. Osteoarthritis Cartilage. 2008;16:1101-9.

28. Honsawek S, Chayanupatkul M. Correlation of plasma and synovial fluid adiponectin with knee osteoarthritis severity. Arch Med Res. 2010;41:593-8.

29. Klein-Wieringa IR, Andersen SN, Herb-van Toorn L, Kwekkeboom JC, van der Helm-van Mil AHM, Meulenbelt I, et al. Are baseline high molecular weight adiponectin levels associated with radiographic progression in rheumatoid arthritis and osteoarthritis? J Rheumatol. 2014;41:853-7.

30. Yusuf E, loan-Facsinay A, Bijsterbosch J, Klein-Wieringa I, Kwekkeboom J, Slagboom PE, et al. Association between leptin, adiponectin and resistin and long-term progression of hand osteoarthritis. Ann Rheum Dis. 2011;70: $1282-4$.

31. Gandhi R, Takahashi M, Virtanen C, Syed K, Davey JR, Mahomed NN. Microarray analysis of the infrapatellar fat pad in knee osteoarthritis: relationship with joint inflammation. J Rheumatol. 2011;38:1966-72.

32. Zhang Z, Xing X, Hensley G, Chang LW, Liao W, Abu-Amer Y, et al. Resistin induces expression of proinflammatory cytokines and chemokines in human articular chondrocytes via transcription and messenger RNA stabilization. Arthritis Rheum. 2010;62:1993-2003.

33. Choe JY, Bae J, Jung HY, Park SH, Lee HJ, Kim SK. Serum resistin level is associated with radiographic changes in hand osteoarthritis: cross-sectional study. Joint Bone Spine. 2012;79:160-5.

34. Roemer FW, Guermazi A, Zhang Y, Yang M, Hunter DJ, Crema MD, et al. Hoffa's fat pad: evaluation on unenhanced MR images as a measure of patellofemoral synovitis in osteoarthritis. AJR Am J Roentgenol. 2009;192: 1696-700

\section{Submit your next manuscript to BioMed Central and we will help you at every step:}

- We accept pre-submission inquiries

- Our selector tool helps you to find the most relevant journal

- We provide round the clock customer support

- Convenient online submission

- Thorough peer review

- Inclusion in PubMed and all major indexing services

- Maximum visibility for your research

Submit your manuscript at www.biomedcentral.com/submit

) Biomed Central 